\title{
LOCAL FRACTIONAL VARIATIONAL ITERATION METHOD FOR SOLVING VOLTERRA INTEGRO-DIFFERENTIAL EQUATIONS WITHIN LOCAL FRACTIONAL OPERATORS
}

\author{
Ammar Ali Neamah \\ Kufa University, Faculty of Computer Science and Mathematics, Najaf, Iraq
}

Received 2014-05-15; Revised 2014-05-27; Accepted 2014-08-20

\begin{abstract}
The paper uses the Local fractional variational Iteration Method for solving the second kind Volterra integro-differential equations within the local fractional integral operators. The analytical solutions within the non-differential terms are discussed. Some illustrative examples will be discussed. The obtained results show the simplicity and efficiency of the present technique with application to the problems for the integral equations.
\end{abstract}

Keywords:Local Fractional Variational Iteration Method, Local Fractional Operator, Local Fractional Volterra Integro-Differential Equation

\section{INTRODUCTION}

The theory of local fractional calculus is one of useful tools to process the fractal and continuously non differentiable functions (Kolwankar and Gangal, 1998; He, 2011; He et al., 2012; Parvate and Gangal, 2009; Carpinteri et al., 2004; Yang, 2011a; 2011b; 2011c). It was successfully applied in local fractional FokkerPlanck equation (Kolwankar and Gangal, 1998), the fractal heat conduction equation (He, 2011; Yang, 2011c), fractal-time dynamical systems (Parvate and Gangal, 2009), fractal elasticity (Carpinteri et al., 2004), local fractional diffusion equation (Yang, 2011c), local fractional Laplace equation (Yang, 2011b; 2012a), local fractional integral equations (Yang, 2012b; 2012c; 2012d), local fractional differential equations (Yang, 2012e; Zhong et al., 2012; Zhong and Gao, 2011), fractal wave equation (Yang, 2011b; 2012a; Yang and Baleanu, 2012).

Recently, the local fractional variational iteration method (Yang and Baleanu, 2012) is derived from local fractional operators (Yang, 2011a; 2011b; 2011c; 2012a; 2012b; 2012c; 2012d; 2012e; Zhong et al., 2012; Zhong and Gao, 2011). The method, which accurately computes the solutions in a local fractional series form or in an exact form, presents interest to applied sciences for problems where the other methods cannot be applied properly.

This study is organized as follows. In section 2 , the basic mathematical tools are reviewed. Section 3 presents the local fractional variational iteration method based on local fractional operator. Illustrative examples is shown in section 4. Conclusions are in section 5.

\section{PRELIMINARY DEFINITIONS}

In this section, we recall briefly some basic theory of local fractional calculus and for more details, (Yang and Baleanu, 2012; Su et al., 2013; Yang et al., 2013a; 2013b; 2013c; Yang, 2012f; Wang et al., 2014; Yang et al., 2013d; Kilbas et al., 2006; Ma et al., 2013; Yang et al., 2013e; 2013f; 2013g).

\section{Definition 1}

Suppose that there is the relation Equation 2.1:

$$
\left|f(x)-f\left(x_{0}\right)\right|<\varepsilon^{\alpha}, 0<\alpha \leq 1
$$


With $\left|x-x_{0}\right|<\delta$, for $\varepsilon, \delta>0$ and $\varepsilon, \delta \in R$, then the function $f(x)$ is called local fractional continuous at $x=x_{0}$ and it is denoted by $\lim _{x \rightarrow x_{0}} f(x)=f\left(x_{0}\right)$.

\section{Definition 2}

Suppose that the function $f(x)$ satisfies condition (2.1), for $x \in(a, b)$; it is so called local fractional continuous on the interval $(a, b)$, denoted by $f(x) \in C_{\alpha}(a, b)$.

\section{Definition 3}

In fractal space, let $f(x) \in C_{\alpha}(a, b)$, local fractional derivative of $f(x)$ of order $\alpha$ at $x=x_{0}$ is given by Equation 2.2:

$$
\begin{aligned}
& D_{x}^{\alpha} f\left(x_{0}\right)=\left.\frac{d^{\alpha}}{d x^{\alpha}} f(x)\right|_{x=x_{0}} \\
& =f^{(\alpha)}(x)=\lim _{x \rightarrow x_{0}} \frac{\Delta^{\alpha}\left(f(x)-f\left(x_{0}\right)\right)}{\left(x-x_{0}\right)^{\alpha}}
\end{aligned}
$$

where, $\Delta^{\alpha}\left(f(x)-f\left(x_{0}\right)\right) \cong \Gamma(\alpha+1) \Delta\left(f(x)-f\left(x_{0}\right)\right)$.

Local fractional derivative of high order is written in the form Equation 2.3:

$$
f^{(k \alpha)}(x)=\frac{d^{k \alpha}}{d x^{k \alpha}} f(x)=\overbrace{D_{x}^{\alpha} D_{x}^{\alpha} \ldots \ldots . D_{x}^{\alpha}}^{\text {ktimes }} f(x) .
$$

\section{Definition 4}

A partition of the interval $[a, b]$ is denoted as $\left(t_{j}, t_{j+1}\right), j=0, \ldots, N-1, t_{0}=a \quad$ and $\quad t_{N}=b \quad$ with $\Delta t_{j}=t_{j+1}-t_{j}$ and $\Delta t=\max \left\{\Delta t_{0}, \Delta t_{1}, \ldots ..\right\}$. Local fractional integral of $f(x)$ in the interval $[a, b]$ is given by Equation 2.4:

$$
\begin{aligned}
& { }_{a} I_{b}^{(\alpha)} f(x)=\frac{1}{\Gamma(1+\alpha)} \int_{a}^{b} f(t)(d t)^{\alpha} \\
& =\frac{1}{\Gamma(1+\alpha)} \lim _{\Delta t \rightarrow 0} \sum_{j=0}^{N-1} f\left(t_{j}\right)\left(\Delta t_{j}\right)^{\alpha}
\end{aligned}
$$

Note: If the functions are local fractional continuous then the local fractional derivatives and integrals exist.
Some properties of local fractional derivative and integrals are given in (Yang, 2012f).

\section{Definition 5}

In fractal space, the Mittage Leffler function, sine function and cosine function are, respectively Equation 2.5 to 2.7:

$$
\begin{aligned}
& E_{\alpha}\left(x^{\alpha}\right)=\sum_{k=0}^{\infty} \frac{x^{k \alpha}}{\Gamma(1+k \alpha)}, 0<\alpha \leq 1 \\
& \sin _{\alpha}\left(x^{\alpha}\right)=\sum_{k=0}^{\infty}(-1)^{k} \frac{x^{(2 k+1) \alpha}}{\Gamma[1+(2 k+1) \alpha]}, 0<\alpha \leq 1 \\
& \cos _{\alpha}\left(x^{\alpha}\right)=\sum_{k=0}^{\infty}(-1)^{k} \frac{x^{2 k \alpha}}{\Gamma[1+2 k \alpha]}, 0<\alpha \leq 1
\end{aligned}
$$

\section{ANALYSIS OF THE METHOD}

The standard $k a$ order local fractional Volterra integrodifferential equation of the second kind is given by:

$u^{(k \alpha)}(x)=f(x)+\frac{1}{\Gamma(1+\alpha)} \int_{0}^{x} K(x, t) u(t)(d t)^{\alpha}$

where,

$$
u^{(k \alpha)}(x)=\frac{d^{k \alpha} u(x)}{d x^{k \alpha}}
$$

$$
u(0)=a_{0}, u^{(\alpha)}(0)=a_{1}, u^{(2 \alpha)}(0)=a_{2}, \ldots, u^{((k-1) \alpha)}(0)=a_{k-1} \text { ar }
$$
e the initial conditions.

According to the rule of local fractional variational iteration method, the correction local fractional functional for Equation 3.1 is given by Equation 3.2:

$$
\begin{aligned}
& u_{n+1}(x)=u_{n}(x) \\
& +\frac{1}{\Gamma(1+\alpha)} \int_{0}^{x} \frac{\lambda(\zeta)^{\alpha}}{\Gamma(1+\alpha)}\left[\int_{0}^{u_{n}^{(k \alpha)}(\zeta)-f(\zeta)-\frac{1}{\Gamma(1+\alpha)}} \zeta_{(\zeta, r) u_{n}(r)(d r)^{\alpha}}\right](d \zeta)^{\alpha}(3
\end{aligned}
$$

where, $\frac{\lambda(\zeta)^{\alpha}}{\Gamma(1+\alpha)}$ is a general fractal Lagrange's multiplier.

Here, we can construct a correction functional as follows Equation 3.3: 
$u_{n+1}(x)=u_{n}(x)+\frac{1}{\Gamma(1+\alpha)} \int_{0}^{x} \frac{\lambda(\zeta)^{\alpha}}{\Gamma(1+\alpha)}\left[u_{n}^{(k \alpha)}(\zeta)-f(\zeta)-\frac{1}{\Gamma(1+\alpha)} \int_{0}^{\zeta} K(\zeta, r) \tilde{u}_{n}(r)(d r)^{\alpha}\right](d \zeta)^{\alpha}$

where, $\tilde{u}_{n}$ is considered as a restricted local fractional variation; that is, $\delta^{\alpha} \tilde{u}_{n}=0$, we obtain the following fractal Lagrange multiplier Equation 3.4:

$$
\frac{\lambda(\zeta)^{\alpha}}{\Gamma(1+\alpha)}=(-1)^{k} \frac{(\zeta-x)^{(k-1) \alpha}}{\Gamma[1+(k-1) \alpha]}
$$

Therefore Equation 3.5 and 3.6:

$$
\begin{aligned}
& u_{0}(x)=u(0)+\frac{x^{\alpha}}{\Gamma(1+\alpha)} u^{(\alpha)}(0)+\cdots \\
& +\frac{x^{(k-1) \alpha}}{\Gamma[1+(k-1) \alpha]} u^{((k-1) \alpha)} \\
& u_{n+1}(x)=u_{n}(x) \\
& +\frac{1}{\Gamma(1+\alpha)} \int_{0}^{x}(-1)^{k} \frac{(\zeta-x)^{(k-1) \alpha}}{\Gamma(1+(k-1))} \\
& {\left[\begin{array}{l}
u_{n}^{(k \alpha)}(\zeta)-f(\zeta)-\frac{1}{\Gamma(1+\alpha)} \\
\int_{0}^{\zeta} K(\zeta, r) u_{n}(r)(d r)^{\alpha}
\end{array}(d \zeta)^{\alpha}, n \geq 0 .\right.}
\end{aligned}
$$

Finally, the solution is Equation 3.7:

$$
u(x)=\lim _{n \rightarrow \infty} u_{n}(x)
$$

\section{ILLUSTRATIVE EXAMPLES}

In this section three examples for the local fractional Volterra integro-differential equation is presented in order to demonstrate the simplicity and the efficiency of the above method.

\section{Example 1}

We consider the local fractional Volterra integrodifferential Equation 4.1:

$$
u^{(\alpha)}(x)=1+\frac{1}{\Gamma(1+\alpha)} \int_{0}^{x} u(t)(d t)^{\alpha}, u(0)=1
$$

The correction functional for this Equation 4.2 is given by:

$$
\begin{aligned}
& u_{n+1}(x)=u_{n}(x)-\frac{1}{\Gamma(1+\alpha)} \int_{0}^{x} \\
& {\left[u_{n}^{(\alpha)}(\zeta)-1-\frac{1}{\Gamma(1+\alpha)} \int_{0}^{\zeta} u_{n}(r)(d r)^{\alpha}\right](d \zeta)^{\alpha}}
\end{aligned}
$$

where, we used $\frac{\lambda(\zeta)^{\alpha}}{\Gamma(1+\alpha)}=-1$ for first-order integrodifferential equation as shown in (3.4).

We can use the initial condition to select $u_{0}(x)=$ $u(0)=1$. Using this selection into the correction functional gives the following successive approximations Equation 4.3 to 4.7:

$$
\begin{aligned}
& u_{0}(x)=1 \\
& u_{1}(x)=u_{0}(x) \\
& -\frac{1}{\Gamma(1+\alpha)} \int_{0}^{x}\left[-\frac{1}{\Gamma(1+\alpha)} \int_{0}^{u_{0}^{(\alpha)}(\zeta)-1} u_{0}(r)(d r)^{\alpha}\right](d \zeta)^{\alpha} \\
& =1+\frac{x^{\alpha}}{\Gamma(1+\alpha)}+\frac{x^{2 \alpha}}{\Gamma(1+2 \alpha)}
\end{aligned}
$$

$u_{2}(x)=u_{1}(x)$

$$
-\frac{1}{\Gamma(1+\alpha)} \int_{0}^{x}\left[\begin{array}{l}
u_{1}^{(\alpha)}(\zeta)-1 \\
-\frac{1}{\Gamma(1+\alpha)} \int_{0}^{\zeta} u_{1}(r)(d r)^{\alpha}
\end{array}\right](d \zeta)^{\alpha}
$$

$$
=1+\frac{x^{\alpha}}{\Gamma(1+\alpha)}+\frac{x^{2 \alpha}}{\Gamma(1+2 \alpha)}+\frac{x^{3 \alpha}}{\Gamma(1+3 \alpha)}+\frac{x^{4 \alpha}}{\Gamma(1+4 \alpha)}
$$$$
u_{3}(x)=u_{2}(x)
$$

$$
\begin{aligned}
& -\frac{1}{\Gamma(1+\alpha)} \int_{0}^{x}\left[\begin{array}{c}
u_{2}^{(\alpha)}(\zeta)-1 \\
-\frac{1}{\Gamma(1+\alpha)} \int_{0}^{\zeta} u_{2}(r)(d r)^{\alpha}
\end{array}\right](d \zeta)^{\alpha} \\
& =1+\frac{x^{\alpha}}{\Gamma(1+\alpha)}+\frac{x^{2 \alpha}}{\Gamma(1+2 \alpha)}+\frac{x^{3 \alpha}}{\Gamma(1+3 \alpha)} \\
& +\frac{x^{4 \alpha}}{\Gamma(1+4 \alpha)}+\frac{x^{5 \alpha}}{\Gamma(1+5 \alpha)}+\frac{x^{6 \alpha}}{\Gamma(1+6 \alpha)}
\end{aligned}
$$

JMSS 
And so on:

$$
\begin{aligned}
& u_{n}(x)=1+\frac{x^{\alpha}}{\Gamma(1+\alpha)}+\frac{x^{2 \alpha}}{\Gamma(1+2 \alpha)} \\
& +\frac{x^{3 \alpha}}{\Gamma(1+3 \alpha)}+\cdots+\frac{x^{2 n \alpha}}{\Gamma(1+2 n \alpha)} \\
& u(x)=\lim _{n \rightarrow \infty} u_{n}(x)=1+\frac{x^{\alpha}}{\Gamma(1+\alpha)} \\
& +\frac{x^{2 \alpha}}{\Gamma(1+2 \alpha)}+\frac{x^{3 \alpha}}{\Gamma(1+3 \alpha)}+\frac{x^{4 \alpha}}{\Gamma(1+4 \alpha)}+\cdots
\end{aligned}
$$

That gives the exact solution Equation 4.8:

$$
u(x)=E_{\alpha}\left(x^{\alpha}\right)
$$

\section{Example 2}

We consider the local fractional Volterra integrodifferential Equation 4.9:

$$
\begin{aligned}
& u^{(2 \alpha)}(x)=1 \\
& +\frac{1}{\Gamma(1+\alpha)} \int_{0}^{x} \frac{(x-t)^{\alpha}}{\Gamma(1+\alpha)} u(t)(d t)^{\alpha}, u(0)=1, u^{(\alpha)}(0)=0 . \\
& u_{1}(x)=u_{0}(x)+\frac{1}{\Gamma(1+\alpha)} \int_{0}^{x} \frac{(\zeta-x)^{\alpha}}{\Gamma(1+\alpha)}\left[u_{0}^{(2 \alpha)}(\zeta)-1-\frac{1}{\Gamma(1+\alpha)} \int_{0}^{\zeta} \frac{(\zeta-r)^{\alpha}}{\Gamma(1+\alpha)} u_{0}(r)(d r)^{\alpha}\right](d \zeta)^{\alpha} \\
& =1+\frac{x^{2 \alpha}}{\Gamma(1+2 \alpha)}+\frac{x^{4 \alpha}}{\Gamma(1+4 \alpha)} \\
& u_{2}(x)=u_{1}(x)+\frac{1}{\Gamma(1+\alpha)} \int_{0}^{x} \frac{(\zeta-x)^{\alpha}}{\Gamma(1+\alpha)}\left[u_{1}^{(2 \alpha)}(\zeta)-1-\frac{1}{\Gamma(1+\alpha)} \int_{0}^{\zeta} \frac{(\zeta-r)^{\alpha}}{\Gamma(1+\alpha)} u_{1}(r)(d r)^{\alpha}\right](d \zeta)^{\alpha} \\
& =1+\frac{x^{2 \alpha}}{\Gamma(1+2 \alpha)}+\frac{x^{4 \alpha}}{\Gamma(1+4 \alpha)}+\frac{x^{6 \alpha}}{\Gamma(1+6 \alpha)}+\frac{x^{8 \alpha}}{\Gamma(1+8 \alpha)} \\
& u_{3}(x)=u_{2}(x)+\frac{1}{\Gamma(1+\alpha)} \int_{0}^{x} \frac{(\zeta-x)^{\alpha}}{\Gamma(1+\alpha)}\left[u_{2}^{(2 \alpha)}(\zeta)-1-\frac{1}{\Gamma(1+\alpha)} \int_{0}^{\zeta} \frac{(\zeta-r)^{\alpha}}{\Gamma(1+\alpha)} u_{2}(r)(d r)^{\alpha}\right](d \zeta)^{\alpha} \\
& =1+\frac{x^{2 \alpha}}{\Gamma(1+2 \alpha)}+\frac{x^{4 \alpha}}{\Gamma(1+4 \alpha)}+\frac{x^{6 \alpha}}{\Gamma(1+6 \alpha)}+\frac{x^{8 \alpha}}{\Gamma(1+8 \alpha)}+\frac{x^{10 \alpha}}{\Gamma(1+10 \alpha)}+\frac{x^{12 \alpha}}{\Gamma(1+12 \alpha)}
\end{aligned}
$$
the correction functional gives the following successive approximations: Equation 4.11 to 4.15:

And so on: 


$$
\begin{aligned}
& u_{n}(x)=1+\frac{x^{2 \alpha}}{\Gamma(1+2 \alpha)}+\frac{x^{4 \alpha}}{\Gamma(1+4 \alpha)}+\frac{x^{6 \alpha}}{\Gamma(1+6 \alpha)}+\cdots+\frac{x^{4 n \alpha}}{\Gamma(1+4 n \alpha)} \\
& u(x)=\lim _{n \rightarrow \infty} u_{n}(x) \\
& =1+\frac{x^{2 \alpha}}{\Gamma(1+2 \alpha)}+\frac{x^{4 \alpha}}{\Gamma(1+4 \alpha)}+\frac{x^{6 \alpha}}{\Gamma(1+6 \alpha)}+\frac{x^{8 \alpha}}{\Gamma(1+8 \alpha)}+\cdots
\end{aligned}
$$

That gives the exact solution Equation 4.16:

$$
u(x)=\cosh _{\alpha}\left(x^{\alpha}\right)
$$

\section{Example 3}

We consider the local fractional Volterra integro-differential Equation 4.17 and 4.18:

$$
u^{(3 \alpha)}(x)=1+\frac{x^{\alpha}}{\Gamma(1+\alpha)}+\frac{x^{3 \alpha}}{\Gamma(1+3 \alpha)}+\frac{1}{\Gamma(1+\alpha)} \int_{0}^{x} \frac{(x-t)^{\alpha}}{\Gamma(1+\alpha)} u(t)(d t)^{\alpha}, u(0)=1, u^{(\alpha)}(0)=0, u^{(2 \alpha)}(0)=1
$$

The correction functional for this equation is given by:

$$
\begin{aligned}
u_{n+1}(x) & =u_{n}(x)-\frac{1}{\Gamma(1+\alpha)} \\
& \int_{0}^{x} \frac{(\zeta-x)^{2 \alpha}}{\Gamma(1+2 \alpha)}\left[u_{n}^{(3 \alpha)}(\zeta)-1-\frac{\zeta^{\alpha}}{\Gamma(1+\alpha)}-\frac{\zeta^{3 \alpha}}{\Gamma(1+3 \alpha)}-\frac{1}{\Gamma(1+\alpha)} \int_{0}^{\zeta} \frac{(\zeta-r)^{\alpha}}{\Gamma(1+\alpha)} u_{n}(r)(d r)^{\alpha}\right](d \zeta)^{\alpha}
\end{aligned}
$$

where, we used $\frac{\lambda(\zeta)^{\alpha}}{\Gamma(1+\alpha)}=-\frac{(\zeta-x)^{2 \alpha}}{\Gamma(1+2 \alpha)}$ for third-order integro-differential equation as shown in (3.4).

Now, we have Equation 4.19 to 4.22:

$$
u_{0}(x)=u(0)+\frac{x^{\alpha}}{\Gamma(1+\alpha)} u^{(\alpha)}(0)+\frac{x^{2 \alpha}}{\Gamma(1+2 \alpha)} u^{(2 \alpha)}(0)=1+\frac{x^{2 \alpha}}{\Gamma(1+2 \alpha)}=1+\frac{x^{2 \alpha}}{\Gamma(1+2 \alpha)}
$$

$u_{1}(x)=u_{0}(x)$

$$
\begin{aligned}
& -\frac{1}{\Gamma(1+\alpha)} \int_{0}^{x} \frac{(\zeta-x)^{2 \alpha}}{\Gamma(1+2 \alpha)}\left[u_{0}^{(3 \alpha)}(\zeta)-1-\frac{\zeta^{\alpha}}{\Gamma(1+\alpha)}-\frac{\zeta^{3 \alpha}}{\Gamma(1+3 \alpha)}-\frac{1}{\Gamma(1+\alpha)} \int_{0}^{\zeta} \frac{(\zeta-r)^{\alpha}}{\Gamma(1+\alpha)} u_{0}(r)(d r)^{\alpha}\right](d \zeta)^{\alpha} \\
& =1+\frac{x^{2 \alpha}}{\Gamma(1+2 \alpha)}+\frac{x^{3 \alpha}}{\Gamma(1+3 \alpha)}+\frac{x^{4 \alpha}}{\Gamma(1+4 \alpha)}+\frac{x^{5 \alpha}}{\Gamma(1+5 \alpha)}+\frac{x^{6 \alpha}}{\Gamma(1+6 \alpha)}+\frac{x^{7 \alpha}}{\Gamma(1+7 \alpha)} \\
& u_{2}(x)=u_{1}(x) \\
& -\frac{1}{\Gamma(1+\alpha)} \int_{0}^{x} \frac{(\zeta-x)^{2 \alpha}}{\Gamma(1+2 \alpha)}\left[u_{1}^{(3 \alpha)}(\zeta)-1-\frac{\zeta^{\alpha}}{\Gamma(1+\alpha)}-\frac{\zeta^{3 \alpha}}{\Gamma(1+3 \alpha)}-\frac{1}{\Gamma(1+\alpha)} \int_{0}^{\zeta} \frac{(\zeta-r)^{\alpha}}{\Gamma(1+\alpha)} u_{1}(r)(d r)^{\alpha}\right](d \zeta)^{\alpha} \\
& =1+\frac{x^{2 \alpha}}{\Gamma(1+2 \alpha)}+\frac{x^{3 \alpha}}{\Gamma(1+3 \alpha)}+\frac{x^{4 \alpha}}{\Gamma(1+4 \alpha)}+\frac{x^{5 \alpha}}{\Gamma(1+5 \alpha)}+\frac{x^{6 \alpha}}{\Gamma(1+6 \alpha)}+\frac{x^{7 \alpha}}{\Gamma(1+7 \alpha)}+\cdots \\
& \text { PlP Science Publications }
\end{aligned}
$$


And so on:

$$
\begin{aligned}
& u_{n}(x)=1+\frac{x^{\alpha}}{\Gamma(1+\alpha)}+\frac{x^{2 \alpha}}{\Gamma(1+2 \alpha)}+\frac{x^{3 \alpha}}{\Gamma(1+3 \alpha)}+\cdots \\
& +\frac{x^{n \alpha}}{\Gamma(1+n \alpha)}-\frac{x^{\alpha}}{\Gamma(1+\alpha)} \\
& u(x)=\lim _{n \rightarrow \infty} u_{n}(x) \\
& =1+\frac{x^{\alpha}}{\Gamma(1+\alpha)}+\frac{x^{2 \alpha}}{\Gamma(1+2 \alpha)}+\frac{x^{3 \alpha}}{\Gamma(1+3 \alpha)}+\cdots-\frac{x^{\alpha}}{\Gamma(1+\alpha)}
\end{aligned}
$$

That gives the exact solution Equation 4.23:

$$
u(x)=E_{\alpha}\left(x^{\alpha}\right)-\frac{x^{\alpha}}{\Gamma(1+\alpha)} .
$$

\section{CONCLUSION}

In this study the Volterra integro-differential equations within the local fractional differential operator had been analyzed using the local fractional variational iteration method. The non-differentiable solutions are obtained. The present method is a powerful tool for solving many integral equations within the local fractional derivatives.

\section{ACKNOWLEDGMENT}

The researchers are grateful to the referees for their invaluable suggestions and comments for the improvement of the paper.

\section{REFERENCES}

Carpinteri, A., B. Cornetti and K.M. Kolwankar, 2004. Calculation of the tensile and flexural strength of disordered materials using fractional calculus. Chaos, Solitons, Fractals, 21: 623 632. DOI: 10.1016/j.chaos.2003.12.081

He, J.H., 2011. A new fractal derivation. Thermal Sci., 15: 145-147.

He, J.H., S.K. Elagan and Z.B. Li, 2012. Geometrical explanation of the fractional complex transform and derivative chain rule for fractional calculus. Phy. Lett. A, 376: 257 259. DOI: 10.1016/j.physleta.2011.11.030

Kilbas, A.A., H.M. Srivastava and J.J. Trujillo, 2006. Theory and Applications of Fractional Differential Equations. 1st Edn., Elsevier, Boston, ISBN-10: 0444518320, pp: 523.
Kolwankar, K. and A.D. Gangal, 1998. Local fractional Fokker-Planck equation. Phys. Rev. Lett., 80: 214217. DOI: $10.1103 /$ PhysRevLett.80.214

Ma, X.J., H.M. Srivastava, D. Baleanu and X.J. Yang, 2013. A new Neumann series method for solving a family of local fractional Fredholm and Volterra integral equations. Math. Problems Eng., 2013: 325121-325126. DOI: 10.1155/2013/325121

Parvate, A. and A.D. Gangal, 2009. Calculus on fractal subsets of real line-I: formulation. Fractals, 17: 5381. DOI: 10.1142/S0218348X09004181

Su, W.H. D. Baleanu, X.J. Yang and H. Jafari, 2013. Damped wave equation and dissipative wave equation in fractal strings within the local fractional variational iteration method. Fixed Point Theory Applic., 2013: 89-89. DOI: 10.1186/1687-18122013-89

Wang, S.Q., Y.J. Yang and H.K. Jassim, 2014. Local fractional function decomposition method for solving inhomogeneous wave equations with local fractional derivative. Abstract Applied Anal., 2014: 176395-176401. DOI: 10.1155/2014/176395

Yang, X.J., 2011a. Local fractional integral transforms. Progr. Nonlinear Sci., 4: 1-225.

Yang, X.J., 2011b. Local Fractional Functional Analysis and its Applications. 1st Edn., Asian Academic Publisher Limited, Hong Kong, ISBN-10: 9881913217, pp: 238.

Yang, X.J., 2011c. Applications of local fractional calculus to engineering in fractal time-space: Local Fractional Differential Equations with local fractional derivative. China University of Mining and Technology.

Yang, X.J., 2012a. Local fractional partial differential equations with fractal boundary problems. ACMA, 1: 60-63.

Yang, X.J., 2012b. Local fractional Kernel transform fractal space and its applications. ACMA, 1: 86-93.

Yang, X.J., 2012c. Local fractional variational iteration method and its algorithms. ACMA, 1: 139-145.

Yang, X.J., 2012d. Local fractional integral equations and their applications. ACSA, 1: 234- 239.

Yang, X.J., 2012e. Local fractional calculus and its applications. Proceedings of the 5th IFAC Workshop Fractional Differentiation and its Applications, (FDA' 12), Nanjing, pp: 1-8.

Yang, X.J., 2012f. Advanced Local Fractional Calculus and its Applications. 1st Edn., World Science, New York, ISBN-10: 1938576012. 
Yang, X.J. and D. Baleanu, 2012. Fractal heat conduction problem solved by local fractional variation iteration method. Thermal Sci., 2: 625-628. DOI: 10.2298/TSCI121124216Y

Yang, Y.J., D. Baleanu and X.J. Yang, 2013a. A local Fractional Variational iteration method for Laplace equation within local fractional operators. Abstract Applied Anal., 2013: 202650-202655. DOI: $10.1155 / 2013 / 202650$

Yang, X.J., D. Baleanu and W.P. Zhong, 2013b. Approximation solutions for diffusion equation on Cantor time-space. Proc. Romanian Acad. A, 14: 127-133.

Yang, X.J. D. Baleanu and J.A.T. Machado, 2013c. Mathematical aspects of the Heisenberg uncertainty principle within local fractional Fourier analysis. Boundary Value Problems, 2013: 131-146. DOI: 10.1186/1687-2770-2013-131

Yang, A.M., X.J. Yang and Z.B. Li, 2013d. Local fractional series expansion method for solving wave and diffusion equations on cantor sets. Abstract Applied Anal., 2013: 351057-351062. DOI: $10.1155 / 2013 / 351057$

Yang, X.J., H.M. Srivastava, J.H. He and D. Baleanu, 2013e. Cantor-type cylindrical-coordinate method for differential equations with local fractional derivatives. Phys. Lett. A, 377: 1696-1700. DOI: 10.1016/j.physleta.2013.04.012
Yang, A.M., Z.S. Chen, H.M. Srivastava and X.J. Yang, 2013f. Application of the local fractional series expansion method and the variational iteration method to the Helmholtz equation involving local fractional derivative operators. Abstract Applied Anal., 2013: 259125-259130. DOI: $10.1155 / 2013 / 259125$

Yang, X.J., D. Baleanu, H.M. Srivastava and J.A.T. Machado, 2013g. On local fractional continuous wavelet transform. Abstract Applied Anal., 2013: 725416-725420. DOI: 10.1155/2013/725416

Zhong, W.P. and F. Gao, 2011. Application of the Yang-Laplace transforms to solution to nonlinear fractional wave equation with local fractional derivative. Proceedings of the 3rd International Conference on Computer Technology and Development, (CTD' 11), pp: 209-213.

Zhong, W.P., F. Gao and X.M. Shen, 2012. Applications of Yang-Fourier transform to local Fractional equations with local fractional derivative and local fractional integral, Adv. Mat. Res., 461: 306-310. DOI: 10.4028/www.scientific.net/AMR.461.306 